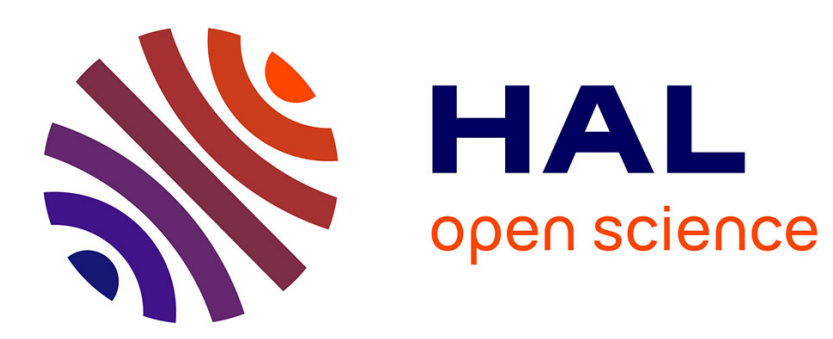

\title{
Comptes rendus de Margot Beal, Des champs aux cuisines. Histoires de la domesticité en Rhône et Loire (1848-1940), ENS Éditions, 2019, 235 p.
}

Clyde Plumauzille

\section{- To cite this version:}

Clyde Plumauzille. Comptes rendus de Margot Beal, Des champs aux cuisines. Histoires de la domesticité en Rhône et Loire (1848-1940), ENS Éditions, 2019, 235 p.. Annales de démographie historique, 2021, n 141 (1), pp.225-253. 10.3917/adh.141.0225 . hal-03495864

\section{HAL Id: hal-03495864 https://hal.science/hal-03495864}

Submitted on 16 Jan 2022

HAL is a multi-disciplinary open access archive for the deposit and dissemination of scientific research documents, whether they are published or not. The documents may come from teaching and research institutions in France or abroad, or from public or private research centers.
L'archive ouverte pluridisciplinaire HAL, est destinée au dépôt et à la diffusion de documents scientifiques de niveau recherche, publiés ou non, émanant des établissements d'enseignement et de recherche français ou étrangers, des laboratoires publics ou privés. 
Clyde Plumauzille, "Compte rendu de Margot Beal, Des champs aux cuisines. Histoires de la domesticité en Rhône et Loire (1848-1940)", Annales de Démographie Historique, 2021.

Margot Beal, Des champs aux cuisines. Histoires de la domesticité en Rhône et Loire (18481940), ENS Éditions, 2019, 235 p.

Issu d'une thèse de doctorat, l'ouvrage de Margot Beal retrace l'histoire de la domesticité rémunérée en France, du milieu du XIXe au milieu du XXe siècle (Beal, 2016). Cette histoire se veut plurielle et située d'où son sous-titre Histoires de la domesticité en Rhône et Loire (1848-1940). Chemin faisant, l'auteure sort l'étude de la domesticité de la capitale parisienne à laquelle elle fut souvent cantonnée et propose de faire jouer les échelles de l'analyse, des grandes villes lyonnaise et stéphanoise aux zones rurales agricoles ou industrielles en passant par tout un maillage de villes moyennes. Par là même, elle entreprend d'étudier de conserve domesticité urbaine et domesticité rurale, la première étant essentiellement une domesticité «à la personne», travaillant au domicile de ceux qui les emploient, la seconde une domesticité «à l'exploitation », travaillant dans les fermes de leurs employeurs. Qu'ils soient cochers, nourrices, bonnes à tout faire, cuisinières, ou valets et bonnes de ferme, ces hommes et ces femmes ont en commun des conditions de travail proches, si ce n'est similaires. Par cette mise en relation, la perspective adoptée par Margot Beal met à mal les fausses évidences de ce qui constituerait « la » domesticité à l'époque de la Révolution industrielle et fait de la définition de la catégorie professionnelle de «domestique » le cœur problématique de son analyse.

Ce parti pris stimulant renoue avec tout un héritage historiographique quelque peu délaissé ces dernières décennies alors que l'histoire de la domesticité peine à constituer un champ de recherche ordonné et institutionnalisé. Longtemps, la discipline historique s'est tenue à distance de cet objet. Réputé « milieu sans histoire », la domesticité ne peut être appréhendée que par bribes, à la faveur d'une documentation souvent « incomplète, émiettée et suspecte » (Guiral et Thuillier, 1978). C'est au tournant des années 1970 et 1980 que les premiers grands travaux sur la «condition domestique » ont vu le jour à la faveur des déplacements du regard historien opérés par la démographie historique, l'histoire sociale et de l'histoire des femmes qui partagent en commun une attention minutieuse aux plus humbles et à leurs modes de vie. Historiens et historiennes s'intéressent alors à souligner la diversité de la " condition servile » notamment selon qu'on soit homme ou femme, le poids et les caractéristiques démographiques des domestiques (comme l'importance du célibat), leur positionnement social ambiguë et leur invisibilisation dans le monde du travail (Zeller, 2016). Ces travaux ont connu un regain dynamique dans les années 2000 à la faveur du Servant Project financé par la Commission Européenne de 2001 à 2004 dont les conclusions mettent notamment en avant l'évolution au 
tournant des XIXe et XXe siècles de la main-d'œuvre domestique européenne, qui se féminise, se prolétarise et provient de plus en plus du milieu rural ${ }^{1}$.

Reprenant le flambeau de ces travaux, et soucieuse de déjouer l'écueil du misérabilisme qui traverse les imaginaires sociaux de cette profession entre service et servitude, l'auteure souhaite à son tour examiner « comment cette catégorie sociale de la domesticité est travaillée par des rapports de pouvoir variés, comment ils interagissent, et comment les domestiques euxmêmes et elles-mêmes se positionnent au sein de ces relations de pouvoir » (p. 12). À travers onze chapitres et un épilogue, Margot Beal s'attelle ainsi à dérouler la complexité de son objet en prenant pour terrain d'investigation les départements de la Loire et du Rhône entre 1848 et 1940. Elle s'appuie pour cela sur des archives administratives qui permettent d'approcher les contours de la population domestique (statistiques et recensements de populations, surveillance de bureaux de placement notamment), des archives privées permettant de retrouver certains de leurs employeurs, et enfin met à profit les archives judiciaires pour documenter les formes de l'expérience domestique et les conflits qui en découlent.

Il s'agit ainsi de poursuivre l'analyse de la domesticité en allant au plus près de ce que constitue le travail domestique et de ce qu'il institue comme rapport de subordination. La brève introduction de l'ouvrage ne permet pas de rendre compte de la méthodologie adoptée face à ces documentations ainsi que le traitement et l'échantillonnage des sources opéré. On pourra se reporter à la thèse et aux annexes intégralement en ligne dont il est issu pour retrouver ce travail de l'archive un peu absent de l'ouvrage, mais indiqué en notes de bas de page. Ainsi, pour les recensements, l'auteure a choisi plusieurs années charnières : 1851-1856, 1876, 1886-1891, 1911, 1921, 1931 et 1936 et procédé à plusieurs échantillonnages. Au total, elle a pu examiner la situation domestique de 87501 habitants de la Loire et du Rhône sur la période 1851-1936, et, parmi elles et eux, 6387 domestiques, travaillant pour 9597 employeurs identifiés (Beal, 2016, 46-49). À noter, l'usage des sources judiciaires fait par l'auteure et plus particulièrement des dossiers de procédures d'assises qui ont été jusqu'alors peu mobilisés pour l'étude des domesticités. Margot Beal se place ici sous les auspices de l'historienne moderniste Carolyn Steedman attentive aux rapports de domination entre employeurs et domestiques et convoquant pour cela les sources de la conflictualité au travail (Steedman, 2009). Au total, 760 affaires sont examinées : 284 pour la période 1848-1876, 283 pour la période 1886-1911 et 193 pour la

\footnotetext{
${ }^{1}$ Servant Project est l'acronyme de « The socio-economic role of males and females domestic service as a factor of European identity ». Ce projet de recherche a été porté par Antoinette Fauve-Chamoux, Raffaella Sarti, Suzy Pasleau et Isabelle Schopp et a fait l'objet de plusieurs publications notamment Suzy Pasleau et Isabelle Schopp, Proceedings of the Servant Project, Presses de l'Université de Liège, 2006, 5 volumes.
} 
période 1915-1936. Ces matériaux, bien que produits dans des circonstances exceptionnelles à l'instar des affaires de vols de domestiques, sont aussi l'occasion d'apprécier le sens que ces hommes et ces femmes donnent à leur travail, sa valeur et l'expérience qu'ils en font. Elles offrent enfin de précieuses informations sur les conditions et les charges de travail, le salaire et les relations entre les domestiques et celles et ceux qui les emploient.

Le premier chapitre dresse un portrait démographique de la domesticité et étudie ses effectifs entre 1848 et 1940 dans le Rhône et la Loire. Trois critères sont retenus pour circonscrire celles et ceux que l'on regroupe sous ce vocable de «domestique » : le fait de se définir comme domestique, la position de subordonné au sein des ménages et l'hébergement au domicile des employeurs. Premier constat, dans les deux départements étudiés, des bourgs ruraux aux grandes villes, la domesticité est omniprésente. Débouché perçu comme naturel et respectable pour toute une jeunesse prolétaire, elle concerne entre 8 et $20 \%$ de la population selon les localités (contre 8 et $15 \%$ en moyenne pour toute la France). La domesticité dite de ferme, essentiellement masculine, y est majoritaire (70\% des domestiques de la Loire et $50 \%$ des domestiques du Rhône). Si la féminisation de la domesticité est patente au XIXe siècle, ce n'est qu'au XXe siècle, avec la baisse de la domesticité de ferme, que la part des femmes dépassera celle des hommes dans ce secteur d'activité. Du fermier à l'aristocrate, en passant par la classe marchande ou les fonctionnaires, différentes classes sociales, ayant commun une certaine aisance financière, recourent à la domesticité. Recensements à l'appui, Margot Beal constate que la classe et le genre jouent un rôle important dans le recrutement de la domesticité. Si les élites économiques sont celles qui recrutent le plus de domestiques, il convient également de noter « la domesticité par foyer est d'autant plus nombreuse qu'il y a d'hommes à servir» (p. 30).

Les chapitres 2 et 3 portent sur la fabrique du corps social des domestiques sous l'effet des politiques publiques et des pratiques patronales. Margot Beal souligne tout d'abord le processus «d'infantilisation des domestiques» (p. 39) qui les place dans une situation de moindre droit. Outre le fait que les domestiques hommes sont exclus dans un premier temps du droit de vote et d'éligibilité, hommes et femmes perdent une partie de leurs droits à ester en justice, à être juré et à témoigner dans certaines circonstances du fait de leur profession. Jusqu'en 1936, ils échappent également à tout un droit du travail qui s'élabore pour encadrer et protéger les travailleurs (journée de douze heures, risques professionnels, repos hebdomadaire). Cette condition professionnelle et civique dégradée facilite l'exploitation et la faible rémunération par le patronat de la main d'œuvre domestique. Qui plus est, lorsque les effectifs de la domesticité agricole baissent à partir des années 1910, l'État met à disposition des 
exploitants des travailleurs juvéniles de l'assistance publique, puis, dans les années 1920, des travailleurs étrangers, notamment polonais. État et patronat partagent en effet le souhait de disposer d'une main d'œuvre abondante et corvéable. Pour les employeurs cette dernière doit être composée d'individus si possible jeunes, ruraux, robustes et surtout célibataires (pour $90 \%$ d'entre eux) afin de favoriser une disponibilité maximale. Les contours de cette main d'œuvre sont très largement naturalisés au motif que certaines personnes seraient « faites » pour servir.

Les chapitres 4 et 5 traitent du quotidien des domestiques et s'appuient plus spécifiquement sur les archives judiciaires pour interroger les rapports de domination rapprochée qui font la spécificité de cette relation de travail. Les domestiques travaillent et habitent le plus souvent au domicile de leurs employeurs. Ce qui peut être perçu comme un avantage économique se révèle également un piège, car cette proximité favorise une mise à disposition totale et chronophage de leur force de travail : « les domestiques sont levés en premier, couchés en dernier » (p. 77). Mais hommes et femmes domestiques ne font pas la même expérience du travail : si les premiers disposent d'une relative liberté de circulation, les secondes en revanche doivent demeurer le plus souvent sous l'étroite surveillance de leurs employeurs. Qui plus est, les rapports de prédation sexuelle sont le lot commun des femmes. L'auteure ne livre pas d'estimation chiffrée, mais souligne que les attentats à la pudeur, les agressions sexuelles ou les viols reviennent régulièrement en marge des procès qu'elle a pu consulter. À partir des années 1920 enfin, leurs conditions de travail connaissent une timide amélioration, permise notamment par l'augmentation des gages et la décohabitation.

Margot Beal aborde ensuite dans les chapitres 6 et 7 la question des modes de gestion et de contrôle développés par le patronat pour faire face à ce qui est perçu comme une « crise de la domesticité » au tournant des XIXe et XXe siècles. Ces derniers s'inscrivent dans la continuité de tout un discours promu par les classes supérieures sur le nécessaire encadrement moral et économique des «classes laborieuses ». Ils s'étendent du contrôle du travail au contrôle sexuel en passant par tout un ensemble de pratiques fondées sur la suspicion et l'infantilisation des domestiques : pièges, fouilles, contrôle de la consommation personnelle. À partir des années 1880, tant le recrutement que la gestion du personnel domestique se professionnalisent. Les bureaux de placement occupent ainsi une position croissante dans la mise en relation de l'offre et de la demande de travail domestique et s'assurent des états de service des individus qui cherchent à se placer. Les nouveaux ménages employeurs, composés des classes moyennes urbaines et de la bourgeoisie entrepreneuriale de la région, adoptent quant à eux une approche de plus en plus rationalisée et standardisée du travail ménager à mesure que ce dernier s'intensifie et que le ratio domestiques/personnes servies diminue. 
Dans les chapitres 8 et 9, il est question des marges d'autonomies ainsi que des compétences physiques, relationnelles et spatiales mises en œuvre par les travailleurs domestiques. Margot Beal mobilise pour cela la notion d'Eigensinn d'Alf Lütke pour restituer ce « sens de soi » qu'ils et elles déploient dans le cadre de leur emploi pour atténuer et négocier les rapports de domination. Les archives judiciaires permettent ainsi d'entrevoir les ruses de ces derniers comme les plaisanteries, les simulations de malaise, de lenteur ou d'incompréhension. Elles dessinent également les contours d'action plus revendicative puisque les domestiques peuvent attaquer leurs employeurs en justice, alerter la police sur d'éventuelles malversations, exposer publiquement les patrons indignes et mauvais payeurs. En dernier recours, ils déguerpissent : «tout au long des affaires rencontrées, hommes et femmes domestiques quittent régulièrement leurs places dans les fermes ou les foyers où le travail est trop dur, les gages peu élevés et la nourriture impropre et insuffisante » (p. 93).

Le livre se conclut sur les transformations qui s'opèrent dans la domesticité dans l'entredeux guerre (chapitre 10) et les relations qu'entretiennent monde ouvrier et monde domestique (chapitre 11). Avec la Grande Guerre et l'appel sous les drapeaux des jeunes hommes domestiques, la réduction et la féminisation de la domesticité se confirment. Parallèlement, la ségrégation professionnelle des classes laborieuses entre domestiques et ouvriers se maintient et se double d'une ségrégation spatiale alors que les premiers se maintiennent en ville et les seconds commencent à peupler les banlieues. Pour autant, la syndicalisation des domestiques, à la faveur notamment du syndicalisme chrétien très présent dans les régions lyonnaise et stéphanoise, ainsi que leur revendication d'appartenance au prolétariat, rapprochent ces derniers du monde ouvrier. Qui plus est, les trajectoires individuelles qui apparaissent à la faveur des archives judiciaires soulignent une importante labilité des parcours professionnels mêlant «temps en domesticité et temps en usine»(p. 198). Outre la reconnaissance des domestiques dans le droit du travail avec l'obtention des congés payés en 1936 au même titre que tous les travailleurs, la décohabitation et l'externalisation grandissante d'une partie du travail domestique contribuent enfin à transformer les cadres du métier et à renforcer la porosité entre prolétariat ouvrier et prolétariat de service.

Au fil de ces différents chapitres, l'ouvrage de Margot Beal apporte une contribution stimulante à l'histoire sociale des domesticités en plaçant la focale au niveau des individus et du périmètre plus ou moins subi de leurs existences. Il mobilise de façon pédagogique toute une épistémologie de la domination pour interroger les rapports de pouvoir - d'âge, de genre et de classe notamment - et les marges de manœuvre qui fondent la condition domestique. La trouvaille des archives judiciaires mobilisées tout au long de l'ouvrage permet quant à elle de 
poser de façon nouvelle la place du droit dans le statut, l'expérience et la saisie du travail des domestiques. Au regard de la richesse de ces sources, l'auteure aurait pu poursuivre l'enquête pour interroger la spécificité du groupe des domestiques plaignants, les arbitrages qui ont conduit à leur passage à la plainte et les résultats qui en découlent tout au long de la période. Rappelons cependant que l'ambition de Margot Beal est d'offrir à un public élargi un récit synthétique sur les domesticités en France à l'orée du XXe siècle, ce qui explique que les données et leur traitement passent parfois au second plan de l'ouvrage, mais n'ôte rien à l'intérêt des perspectives ouvertes et de l'appel à une reconnaissance tant historiographique que sociale des domesticités.

BeAL, MARgOt (2016), La domesticité dans la région lyonnaise et stéphanoise: vers la constitution d'un prolétariat de service (1848-1940) (thèse sous la direction de Laura Downs), Institut universitaire européen de Florence, Florence Consulté à l'adresse http://cadmus.eui.eu//handle/1814/45604.

Guiral, Pierre, Thuillier, GuY (1978), La Vie quotidienne des domestiques en France au XIX $X^{e}$ siècle, Hachette Littérature.

Steedman, Carolyn (2009), Labours Lost: Domestic Service and the Making of Modern England, Cambridge, Cambridge University Press.

ZELLER, OLIVIER (2016), «Revisiter l'histoire française des serviteurs et des domestiques », Revista de Historia Moderna, Anales de la Universidad de Alicante, Revista de Historia Moderna, Anales de la Universidad de Alicante, (34), 257-278. 